\title{
Enlaces Por La Salud: A Personal Health Navigator Intervention Grounded in the Transnational Framework
}

\author{
Lisa Hightow-Weidman ${ }^{1,2}$ (1) $\cdot$ Joaquin Carcano ${ }^{3} \cdot$ Seul Ki Choi $^{4} \cdot$ Lynne Sampson $^{1} \cdot$ Clare Barrington $^{2}$
}

Accepted: 26 March 2021

(c) The Author(s) 2021

\begin{abstract}
Despite the disproportionate burden of HIV among Latinxs, there is a paucity of culturally appropriate interventions that have shown efficacy at increasing their engagement and retention in HIV care. We describe the development and implementation of Enlaces, a six-session, individual-level intervention, guided by the transnational framework, to improve HIV care outcomes for newly diagnosed and out-of-care Mexican men and transgender women (TW). Descriptive statistics summarizing baseline data and implementation outcomes are provided. 91 participants enrolled between October 2014 and August 2017. Intervention engagement and satisfaction was high; $81.3 \%$ completed all six sessions and $100 \%$ were very satisfied/satisfied with their experience. Successful implementation of the ENLACES intervention was the result of establishing client trust and maintaining a flexible, supportive approach to intervention delivery. Use of the transnational framework provided a contextualized approach to engaging with Mexican men and TW living with HIV that can be adapted to other Latino populations.
\end{abstract}

Keywords Latinx $\cdot \mathrm{HIV} \cdot$ Engagement in care $\cdot$ Intervention transnational

\section{Introduction}

In 2017, Hispanic/Latinx (hereafter, Latinx) made up approximately $10 \%$ of North Carolina's (NC) population [1], a $91 \%$ increase since 2004. HIV in NC disproportionately affects Latinx men who have sex with men (MSM) and transgender women (TW). In 2017, eighty-five percent of new HIV cases among Latinx men in NC in 2017 were attributed to male-to-male sex [1]. The rate of HIV infection among Latinx MSM was 830 per 100,000 in 2017, nearly 4 times the rate of White MSM (230 per 100,000) [2]. Among Latinx living with HIV in NC, only $51 \%$ were virally suppressed in 2017, the lowest rate of any racial/ ethnic group [3]. Further, among MSM with HIV in NC,

Lisa Hightow-Weidman

Lisa_hightow@med.unc.edu

1 Institute for Global Health and Infectious Diseases, University of North Carolina, Chapel Hill, USA

2 Gillings School of Global Public Health, University of North Carolina, Chapel Hill, USA

3 The Latino Commission on AIDS, New York, USA

4 School of Nursing, University of Pennsylvania, Philadelphia, USA
$61 \%$ of Latinx MSM are virally suppressed compared to $70 \%$ of white MSM [2]. In 2017, a total of 86 transgender people were estimated to be living with HIV in NC, $15 \%$ of whom are Latinx. This is likely an underestimate given that transgender identity was not routinely captured in the NC surveillance system until 2015, limiting accurate surveillance and measurement [1].

Latinx MSM and TW's access to and engagement in HIV care is shaped by the interplay between factors including immigration and labor policies, intersecting stigma and discrimination, social isolation, and limited access to culturally competent health services [4-8]. Undocumented Latinx immigrants are less likely to have a usual source of care than those who are documented and concerns about job loss for requesting time off as well as fear of deportation from service utilization limits engagement and leads to poor outcomes [9-11].

Qualitative studies conducted with Latinx men and TW in NC underscore the interplay of the migration, HIV vulnerability and the HIV care experiences of this population [12, 13]. Key findings from the formative research conducted to inform the development of the intervention described in the current study include the salience of the mental health burden of diagnosis and the importance of participants' social networks both within the US and country of origin for provision 
of support [14]. Most participants had experienced interruptions in their care due to both intersecting stigmatized identities (e.g. being gay, Latinx, undocumented) and intersecting structures (healthcare, immigration policy, institutionalized homophobia). Undocumented participants directly connected their immigration status to their ability to get work, which then affected their retention in HIV care and treatment adherence [14]. Participants also describe using a range of strategies to exert control over their lives with HIV including: understanding their infection, engaging with health care, and developing relationships with health professionals [15].

Despite recognition of the disproportionate burden of HIV among Latinxs, there is a paucity of culturally appropriate interventions that have shown efficacy at increasing engagement and retention in care for Latinx living with HIV especially for those who identify as sexual or gender minorities [16]. Project STYLE (Strength Through Youth Livin' Empowered), an intervention for Black and Latinx young men who have sex with men living with HIV which included a social marketing campaign targeting youth and members of their sexual and social networks, testing and community outreach, and a tightly linked medical-social support network resulted in increased HIV diagnoses, timely engagement in care and improvements in overall retention rates. However, only $11 \%(n=9)$ of the study sample identified as Latinx [17]. Anti-Retroviral Treatment and Access to Services (ARTAS), an individual-level, multi-session, intervention based on the Strengths-based Case Management (SBCM) model, was found to significantly increase engagement in care at both 6 and 12 months [18]. While the ARTAS intervention was not developed specifically for Latinx populations, in multivariate analysis, the intervention was significantly $(\mathrm{p}<0.05)$ stronger among Latinx participants than other racial/ethnic groups combined [19].

Effective strategies to support engagement in care, adherence to antiretroviral therapy (ART) and viral suppression are needed in new Latinx settlement states, such as NC. To this end, we developed Enlaces por la Salud (hereafter, Enlaces) to improve HIV continuum of care outcomes (e.g. retention in HIV care, viral suppression) for newly diagnosed and out-of-care Mexican men and TW. In this paper, we describe the development and implementation of Enlaces; baseline characteristics of the study sample are provided.

\section{Methods}

\section{Intervention Overview}

We developed Enlaces as part of a 5-year Special Project of National Significance (SPNS) funded by the Health Resources and Services Administration (HRSA). All HRSA grantees developed innovative HIV linkage and engagement program using a transnational approach tailored to either Mexican or Puerto Rican participants. Further, interventions were specifically developed to focus on populations who were newly diagnosed within the past six months or out-ofcare (diagnosed with HIV more than six-months ago with gaps in care $>6$ months). While both US and foreign-born Latinx populations in $\mathrm{NC}$ represent a diversity of cultural/ ethnic origins, the majority of Latinx residents identify Mexican as their primary ancestry [20], thus, Mexico was chosen as the country of origin upon which Enlaces was developed. Development of Enlaces was interdisciplinary, including clinical and public health researchers, community advocates, peer navigators and case managers.

\section{Theoretical Underpinnings}

Enlaces was informed by concepts related to transnationalism, migration and stigma. Transnationalism refers to networks, resources and experiences in both countries of origin and countries of settlement and connections between the two [21]. Transnationalism is enacted via communication, social and economic exchanges, and travel which allow individuals to be connected, engaged, and influenced by two or more communities simultaneously [21]. We also drew upon Zimmerman, Kiss, and Hossain's Migratory Process Framework (MPF) [22], a rights-based model that considers migration to be cyclical and multi-staged, dividing the process into five phases: (1) Pre-departure; (2) Travel; (3) Destination; (4) Interception; and (5) Return. Each of the phases involves distinct health-related risk exposures with cumulative effects over the course of migration, and each also presents distinct opportunities for intervention. The MPF recognizes the transnational experience of migrants across all stages of the framework. In our intervention sessions, we applied the MPF as we considered how stages of migration and migration experiences may influence engagement with HIV care (Table 1). We also applied Parker and Aggleton's conceptualization of HIV-related stigma as the product of multiple, overlapping forms of stigma related to sexuality, gender, class, and race/ethnicity to the intervention content (Table 1) [23].

The core elements of the ARTAS strengths-based case management model informed intervention format (e.g., structured individual sessions with each client) and delivery (e.g., maintaining a client-driven approach and encouraging them to identify and use strengths, abilities and skills to engage in medical care) [18]. Adaptations were made to: (1) ensure all materials were both culturally and linguistically appropriate and acknowledge the transnational perspective; (2) include content relevant for those newly diagnosed as well as those who had fallen out of care; and (3) extend intervention content and duration (from 90 days to 6 months) to address long-term retention and viral suppression. 


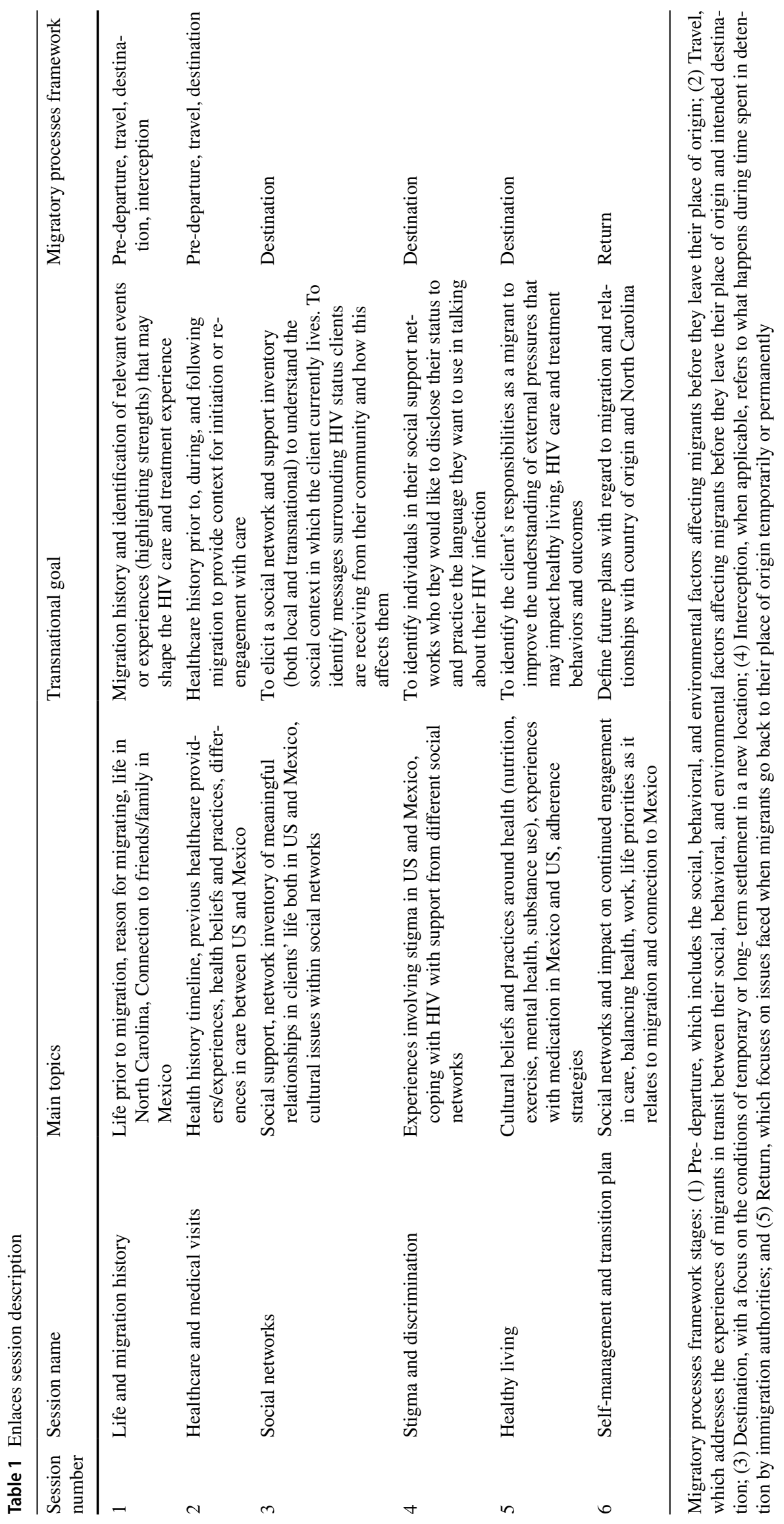




\section{Intervention description}

Enlaces included six-sessions delivered to individuals over six months by two bilingual Personal Health Navigators (PHNs) located in the Raleigh-Durham-Chapel Hill and Charlotte metro areas of NC. Each session was anchored in the transnational framework, starting with participant's migration story, and addressed their experiences with health care and stigma, language preferences, and social networks in both NC and Mexico. Each session had a transnational goal that guided the content and activities. PHNs employed a strengths-based perspective, to provide support, help participants identify individual resources and address previous or anticipated barriers to care and promote long-term retention [24].

Session descriptions. And transnational goals are provided in Table 1. Engagement in the program and Session 1 began with the participant sharing their life story pre and post-migration to the US, if applicable, to operationalize of the transnational approach. This allowed the PHN to have a comprehensive overview of the participant's history and the impact on their current daily life, particularly healthcare management, which laid the groundwork for the ongoing relationship. Important questions included: (1) How are participants still connected to their country of origin?; (2) How does their migration story continue to affect their current life situation?; (3) What has been their experience in the US? Content covered throughout the course of the intervention included HIV diagnosis, appointment debriefs, general HIV knowledge, and working with healthcare teams (Session 2), social networks and support (Session 3), stigma and disclosure (Session 4), health history, healthy living, mental health, and medication adherence (Session 5), and transition plans post-Enlaces (Session 6).

PHNs were required to demonstrate both Spanish and English proficiency and have experience in delivering case management. PHNs underwent multiple Enlaces training, including an initial webinar by The Latino Commission on AIDS. The webinar provided project background, relevant research findings, and an introduction to HIV care provision. This was followed by a 2 day in-person training which focused on the structure of strengths-based case management, how to interact with participants and people of diverse backgrounds, and, reviewing paperwork and the process involved in connecting a participant to social services. Other highlights included demonstrations and role-plays of the role and responsibilities of the PHN, recognizing the principles of a participant -centered, goal setting, and establishing effective community collaborations and relationships for seamless linkage and referrals to care.

\section{Study sample}

Participants were referred to Enlaces between October 2014 through August 2017 from health care providers and staff at HIV care clinics, the state's disease intervention specialists (DIS) and state bridge counselors (SBC) who work with newly diagnosed and out-of-care individuals respectively, area HIV service organizations, and by running periodic (e.g. bimonthly) clinic out-of-care lists and providing these referrals to PHNs. Eligibility criteria were: (1) self-identifying as Mexican/Mexican-American; (2) Male gender assigned at birth; (3) Diagnosed HIV-positive; (4) 18 years or older; (5) Able to provide informed consent; and (6) English or Spanish speaking. Participants had to either be newly diagnosed (within the past 6 months) or out-of-care (diagnosed with HIV more than 6-months ago and not being seen regularly by an HIV primary care provider (e.g. gaps in care $>6$ months). Frequent cancellations and rescheduled appointments as well as use of walk-in clinic hours were used as indicators of inconsistent engagement in care, as determined by a medical chart review. Ten referred clients did not qualify for the study (did not identify as Mexican/ Mexican American, not newly diagnosed or out-of-care). Of the remaining 110 participants who were eligible, eight did not attend the initial enrollment visit and 11 either refused to participate or were unable to be contacted, for a total of 91 enrolled participants. Assuming that the prescreened pool was all eligible $(n=110)$, this study achieved $83 \%$ cooperation.

The study was approved by the Institutional Review Board (IRB) at the University of North Carolina (IRB \#13-3673).

\section{Data Collection}

Upon confirmation of eligibility, participants provided informed consent, which included a Health Insurance Portability and Accountability Act (HIPAA) waiver for release of their medical information related to HIV clinical care. Participants completed surveys at baseline, 6 months and 12 months. Surveys lasted approximately $1-1 \frac{1}{2}$ hours and participants received $\$ 30$ compensation for completion of each survey. A comprehensive quantitative assessment was comprised of measures that were pulled from the literature and when possible, validated and/or published in Spanish. All items in English were translated by a professional Spanish document translator. The full list of items was then reviewed by a team of two native Spanish speakers. Discrepancies were resolved iteratively, including pilot testing the measures with native Spanish speakers at study sites. Surveys were administered using a computer assisted survey instrument in the participants' language of choice (English or Spanish). Online data from the baseline survey 
and intervention satisfaction questionnaire were used for this analysis. All data were stored in a secure server maintained by the HRSA Evaluation and Technical Assistance Center.

\section{Measures}

\section{Demographic and Psychosocial Variables}

Demographic variables included age, gender identity, education, income and relationship status.

Alcohol Use was assessed using the Alcohol Use Disorders Identification Test (AUDIT-C) [25, 26], scored on a scale of $0-12$, with 0 reflecting no alcohol use. In men, a score of 4 or more is considered positive for a substance use disorder. Depression was assessed with the CES-D using 10 questions scored from 0 to $3(0=$ rarely or never to $3=$ most or all of the time). Total scores range from 0 to 30 [27]. Higher scores suggest greater severity of symptoms with scores $\geq 10=$ depressive symptoms.

\section{Transnational Practices, Migration History and Acculturation}

Individual items measured country of origin (COO), years in the US, and first language spoken. Ties to participants $\mathrm{COO}$ was assessed by questions that measured how often they travel to, send money or goods to their COO.

The Bidimensional Acculturation Scale, consisting of three subscales (Language use, Linguistic proficiency and Electronic media) was also used [28]. Answers to 12 items that measure each cultural domain (Latinx and US) were averaged across items for each respondent. A sample item from Language use is: How often do you speak English/ Spanish with your friends? A sample item form Linguistic proficiency is How well do you read English/Spanish? A sample item from Electronic media is How often do you watch television programs in English/Spanish? Each respondent was assigned two scores: (a) one for the average of the 12 items making up the Latinx domain and (b) another score for the 12 items forming the US domain. The possible total score range is from 1 to 4 for each cultural domain. Based on the literature, a score of 2.5 was used as a cutoff score to indicate low or high level of adherence to each cultural domain.

The US cultural identity subscale was based on six items from the American Identity Questionnaire [29]. A parallel six-item subscale was developed to assess Latinx identity as part of the Abbreviated Multidimensional Acculturation Scale (AMAS-ZABB) [30]. Thus, two separate scores are obtained for identity: one for US-American identity and one for Latinx identity. Sample items include: I think of myself as US-American (or Latinx) and being US American (or Latinx) plays an important part in my life. Each were scored on a 4-point Likert scale ( $1=$ strongly disagree, $4=$ strongly agree) with higher scores indicating greater identification with culture of origin.

\section{Intervention Satisfaction}

Participants completed a paper-based questionnaire at 12 months to assess intervention satisfaction. Four questions, rated on a four-point Likert scale from very satisfied to very dissatisfied included "How satisfied are you with your experience participating in the ENLACES intervention?" "How satisfied are you with the services, if any, you were linked to during ENLACES?" "How satisfied are you with the skills you learned and/or enhanced by participating in the intervention?" "How satisfied are you with the Personal Health Navigator you worked with over the course of the intervention?" One question, "Would you recommend ENLACES to anyone you know?" had a dichotomous outcome (yes/no).

\section{Statistical Analysis}

Univariate and bivariate analyses were conducted to describe the population at baseline, based on the categorization of new to care or re-engaging. All data was analyzed using SAS 9.4 (SAS Institute; Cary, North Carolina).

\section{Results}

\section{Sample Demographics}

Baseline characteristics of Enlaces participants by HIV care status is provided in Table 2; 49 were new to care and 42 re-engaging. The majority identified as male (89.9\%). Mean age was 36.8 years and the majority identified as male (89.9\%). Participants reported substantial socioeconomic barriers including high levels of poverty $(60.5 \%$ had a total household income of $<\$ 11,490$ in the prior year), past incarceration (33.0\%) and lack of healthcare coverage (81.3\%). Approximately one-quarter reported being unstably housed in the 6 months prior to study entry. Approximately one-third (30.8\%) of the sample screened positive for an alcohol use disorder and $18.7 \%$ reported use of other substances (noninjection). Compared to participants re-engaging in care, those new to care were about 5 years younger (34.6 years vs. 39.4 years; $p=0.04$ ). There were no other statistically significant differences between the two populations.

\section{Transnational Practices}

Migration history, transnational practices and acculturation are described in Table 3. Most participants were born 
Table 2 Baseline characteristics of enlaces participants by HIV care status

\begin{tabular}{|c|c|c|c|c|}
\hline Variable & Total sample $(n=91)$ & New to care $(n=49)$ & Re-engaging $(n=42)$ & p-value \\
\hline Age, mean $[\mathrm{SD}]$ & 36.8 [10.9] & $34.6[10.5]$ & $39.4[11.0]$ & 0.04 \\
\hline Gender preferred $(n=79)$ & & & & 0.83 \\
\hline Male & $71(89.9 \%)$ & $39(88.6 \%)$ & $32(91.4 \%)$ & \\
\hline Female & $2(2.5 \%)$ & $1(2.3 \%)$ & $1(2.9 \%)$ & \\
\hline Trans-female or transgender & $2(2.5 \%)$ & $2(4.6 \%)$ & $0(0 \%)$ & \\
\hline Other & $4(5.1 \%)$ & $2(4.6 \%)$ & $2(5.7 \%)$ & \\
\hline Highest education & & & & 0.78 \\
\hline Up to 8 th grade & $33(36.3 \%)$ & $20(40.8 \%)$ & $13(30.9 \%)$ & \\
\hline 9th-11th grade & $25(27.5 \%)$ & $13(26.5 \%)$ & $1228.6 \%$ ) & \\
\hline Grade 12 or GED & $16(17.6 \%)$ & $8(16.3 \%)$ & $8(19.1 \%)$ & \\
\hline Some college or above & $17(18.7 \%)$ & $8(16.3 \%)$ & $9(21.4 \%)$ & \\
\hline Relationship status & & & & 0.66 \\
\hline Single & $58(63.7 \%)$ & $30(61.2 \%)$ & $28(66.7 \%)$ & \\
\hline In a relationship & $33(36.3 \%)$ & $19(38.8 \%)$ & $14(33.3 \%)$ & \\
\hline Annual household income & & & & 0.95 \\
\hline$\leq \$ 11,490$ & $55(60.5 \%)$ & $31(63.3 \%)$ & $24(57.1 \%)$ & \\
\hline$>\$ 11,490$ & $36(39.5 \%)$ & $18(36.7 \%)$ & $18(42.9 \%)$ & \\
\hline Ran out of money in $\geq 3$ months out of past 6 & $24(26.4 \%)$ & $11(22.4 \%)$ & $13(30.9 \%)$ & 0.31 \\
\hline Had to borrow money ${ }^{a}$ & $61(67.0 \%)$ & $35(71.4 \%)$ & $26(61.9 \%)$ & 0.38 \\
\hline Sometimes/often hungry ${ }^{\mathrm{a}}$ & $14(15.4 \%)$ & $5(10.2 \%)$ & $9(21.4 \%)$ & 0.35 \\
\hline Unstable housing $^{\mathrm{a}}$ & & & & 0.11 \\
\hline Rarely/sometimes & $19(20.9 \%)$ & $11(22.4 \%)$ & $8(19.1 \%)$ & \\
\hline Often & $4(4.4 \%)$ & $0(0 \%)$ & $4(9.5 \%)$ & \\
\hline Jail/prison (lifetime) & $30(33.0 \%)$ & $17(34.7 \%)$ & $13(30.9 \%)$ & 0.91 \\
\hline Jail/prison ${ }^{\mathrm{a}}$ & $11(36.7 \%)$ & $6(35.3 \%)$ & $5(38.5 \%)$ & 1.00 \\
\hline \multicolumn{5}{|l|}{ Gender identity of sex partners ${ }^{a}$} \\
\hline Male & $43(47.2 \%)$ & $27(55.1 \%)$ & $16(38.1 \%)$ & 0.14 \\
\hline Female & $22(24.2 \%)$ & $10(20.4 \%)$ & $12(28.6 \%)$ & 0.46 \\
\hline Genderqueer & $1(1.1 \%)$ & $0(0 \%)$ & $1(2.4 \%)$ & 0.46 \\
\hline Not had sex & $19(20.9 \%)$ & $10(20.4 \%)$ & $9(21.4 \%)$ & 1.00 \\
\hline No health coverage & $74(81.3 \%)$ & $43(87.8 \%)$ & $31(73.8 \%)$ & 0.11 \\
\hline Proportion with alcohol disorder (AUDIT-C $\geq 8$ ) & $28(30.8 \%)$ & $16(32.6 \%)$ & $12(28.6 \%)$ & 0.82 \\
\hline Non-injection drug use ${ }^{1}$ & $17(18.7 \%)$ & $11(22.5 \%)$ & $6(14.3 \%)$ & 0.66 \\
\hline Depressed (CES-D 10 $\geq 10)$ & $36(40 \%)$ & $21(43.8 \%)$ & $15(35.7 \%)$ & 0.52 \\
\hline
\end{tabular}

${ }^{\text {a }}$ Time frame is past 6 months

in Mexico (87.8\%), spoke Spanish as their primary language $(94.4 \%)$ and immigrated to the US at a mean age of 20.1 years. While participants rarely traveled back to Mexico, most occasionally or regularly sent money (70\%) or goods (45.5\%). Mean scores were higher on the Latinx scales compared to the US Scales, indicating greater identification with Latinx culture and language on both the Bidimensional Acculturation Scale (3.48 vs. 2.36) and cultural identity scales (3.81 vs. 2.30). There were no significant differences related to transnational practices between new to care and re-engaging participants.

\section{Implementation Outcomes and Satisfaction}

Overall sample retention was $78.0 \%(\mathrm{n}=71)$ at 6 months and $79.1 \%(\mathrm{n}=72)$ at 12 months. The majority of participants $(\mathrm{n}=74,81.3 \%)$ completed all six sessions. A third of the sample $(\mathrm{n}=33,36 \%)$ received more than one session in a single encounter, with $23 \%$ of all encounters involving multiple sessions. The mean number of days from the first session to the last was 166 days ( $\sim$ and a half months). Overall satisfaction with the intervention was high $(n=72)$; $100 \%$ were very satisfied/satisfied with their experience in 
Table 3 Transnational practices among enlaces participants

\begin{tabular}{|c|c|c|c|c|}
\hline Variable & Total sample $(n=91)$ & New to care $(n=49)$ & Re-engaging $(n=42)$ & p-value \\
\hline Country born & & & & 1.00 \\
\hline US & $9(10.0 \%)$ & $5(10.4 \%)$ & $4(9.5 \%)$ & \\
\hline Mexico & $79(87.8 \%)$ & $41(85.4 \%)$ & $38(90.5 \%)$ & \\
\hline Other & $2(2.2 \%)$ & $2(4.2 \%)$ & $0(0 \%)$ & \\
\hline Arrival in the United States $(\mathrm{N}=80)$ & & & & 0.15 \\
\hline Mean $[\mathrm{SD}]$ & $20.1[8.8]$ & $18.8[8.1]$ & $21.7[9.4]$ & \\
\hline First spoken language & & & & 0.51 \\
\hline English & $3(3.3 \%)$ & $1(2.1 \%)$ & $2(4.8 \%)$ & \\
\hline Spanish & $85(94.4 \%)$ & $45(93.8 \%)$ & $40(95.2 \%)$ & \\
\hline Both & $2(2.2 \%)$ & $2(4.2 \%)$ & $0(0 \%)$ & \\
\hline \multicolumn{5}{|l|}{ Bidimensional acculturation scale } \\
\hline US: mean [SD] & $2.36[0.85]$ & $2.37[0.89]$ & $2.36[0.79]$ & 0.96 \\
\hline Latinx: mean $[\mathrm{SD}]$ & $3.48[0.56]$ & $3.47[0.61]$ & $3.49[0.51$ & 0.89 \\
\hline \multicolumn{5}{|l|}{ Cultural identity (US) } \\
\hline US: mean [SD] & $2.30[0.95]$ & $2.42[0.99]$ & $2.17[0.90]$ & 0.22 \\
\hline Latinx: mean [SD] & $3.81[0.46]$ & $3.84[0.39]$ & $3.78[0.53]$ & 0.56 \\
\hline How often travel to country of origin (COO) & & & & 0.95 \\
\hline Never/rarely & $79(87.8 \%)$ & $43(89.6 \%)$ & $36(85.7 \%)$ & \\
\hline Sometimes/often & $11(12.2 \%)$ & $5(10.4 \%)$ & $6(14.3 \%)$ & \\
\hline Send money to $\mathrm{COO}$ & & & & 0.09 \\
\hline No & $27(30.0 \%)$ & $18(37.5 \%)$ & $9(21.4 \%)$ & \\
\hline Yes, occasional & $45(50.0 \%)$ & $24(50.0 \%)$ & $21(50.0 \%)$ & \\
\hline Yes, regularly & $18(20.0 \%)$ & $6(12.5 \%)$ & $12(28.6 \%)$ & \\
\hline Send goods to $\mathrm{COO}$ & & & & 0.50 \\
\hline No & $49(54.4 \%)$ & $29(60.4 \%)$ & $20(47.6 \%)$ & \\
\hline Yes, occasional & $29(32.2 \%)$ & $13(27.1 \%)$ & $16(38.1 \%)$ & \\
\hline Yes, regularly & $12(13.3 \%)$ & $6(12.5 \%)$ & $6(14.3 \%)$ & \\
\hline Contact with those in $\mathrm{COO}$ & & & & 0.36 \\
\hline Mean [SD] & $2.49[0.94]$ & $2.41[0.95]$ & $2.59[0.92]$ & \\
\hline
\end{tabular}

the intervention, $98.6 \%$ were very satisfied/satisfied with the services they were linked to during ENLACES, 98.6\% were very satisfied/satisfied with the skills learned and/or enhanced by participating in the intervention, and $100 \%$ were very satisfied/satisfied with the PHN. All participants reported being willing to recommend Enlaces to others in need of similar services.

\section{Discussion}

This paper describes the foundation and development of Enlaces, a HRSA-supported SPNS intervention designed to improve HIV outcomes among Mexican men and TW. Given that HIV rates continue to rise among Latinx populations in the Southern US, novel interventions to facilitate diagnosis, entry and retention in care are urgently needed. Enlaces is, to our knowledge, the first transnational intervention developed to address barriers that Latinx men and TW face along the
HIV care continuum, including stigma, cultural beliefs and practices around health and social networks of support.

Enlaces was developed through a strengths-based perspective; which acknowledged the individual and structural barriers to care faced by participants and prioritized their personal strengths and resiliency to address them. Indeed, at baseline, participants reported experiencing substantial socioeconomic barriers to navigating medical care including high rates of financial insecurity (e.g., low annual incomes, housing and food insecurity) along with notably high rates of drug and alcohol use. Provision of tailored strengthsbased solutions including an assessment and understanding of transnational practices may help to assist participants in successfully navigating these challenges.

Transnationalism was evident among participants, most of whom who maintained strong social, cultural and economic ties with their COO despite living in the US for many years (mean 20.1 years) and traveling back to Mexico rarely or not at all [31, 32]. Higher scores on the Bidimensional 
Acculturation Scale and cultural identity measure indicate that participants tended to have greater identification with their Latinx ethnic identity, living their lives and communicating mostly in Spanish. Consideration of these ties and the spaces they occupy within the daily lives of migrants is crucial for understanding the complex role that transnational networks can play in both promoting and hindering engagement in healthcare and self-management of chronic health conditions $[32,33]$. The impact of transnationalism on the health behaviors of Latinx populations living with HIV has not been fully elucidated; a gap that this project seeks to fill through both quantitative and qualitative analyses that are forthcoming.

We found overall high levels of engagement with both the intervention as well as retention in the study. This may be attributed to our use of the transnational framework, which encouraged a holistic approach and facilitated trust between PHNs and the clients. In addition, PHNs engaged in frequent contact with clients through phone calls and text messages and maintained flexibility with regard to scheduling sessions (e.g., completing multiple sessions during an encounter, conducting sessions during weekends or evening hours). This was critical given that many participants had issues with transportation and maintained work schedules that did not allow time off, including jobs that required them to spent long spans of time working out-of-state.

Future interventions with Latinx populations should consider similar strategies to encourage participation.

While we faced initial difficulties in building referral networks, gaining buy-in from providers and clinics and establishing our program's presence and reputation, we leveraged team member's long-standing history and local trust of our community partners and engaged in visibility campaigns (through radio shows, attending regional conferences, presentations to providers, and numerous meetings with area staff) to inform them of our services and outline mutually beneficial strategies of referrals and care engagement.

\section{Limitations}

While the primary focus of the Enlaces intervention was on Mexican MSM and TW, a decision to enroll all participants born male was made given prior research that found that immigrant sexual minority Latinx men may be reluctant to disclose their sexual orientation [34]. The focus on the subpopulation of Mexicans living in NC may limit the generalizability of our findings given that their HIV related healthcare experiences and barriers and facilitators to care may differ from Latinx populations currently residing in other US states or with different ancestries. Finally, while we do not ask about immigration status, within Enlaces sessions, PHNs explicitly address how fears of deportation, as well as time and economic pressures may exacerbate barriers to care $[35,36]$.

\section{New Contribution to the Literature}

Enlaces is a novel intervention developed to improve HIV continuum of care outcomes for newly diagnosed and out-of-care/inconsistently-in-care Mexican men and TW. Future analyses will determine the effectiveness of Enlaces at increasing engagement in care and viral suppression. Increasing the proportion of Latinx individuals living with HIV who are engaged in care and virally suppressed will help to achieve the national goals of reducing new infections and decreasing health disparities; thus, moving closer towards the ultimate goal of ending the epidemic [37].

Acknowledgements This project was supported by the Health Resources and Services Administration (HRSA) of the U.S. Department of Health and Human Services (HHS) under Grant Number H97HA26505, SPNS Latinx Initiative. This information or content and conclusions are those of the author and should not be construed as the official position or policy of, nor should any endorsements be inferred by HRSA, HHS or the U.S. Government.

Open Access This article is licensed under a Creative Commons Attribution 4.0 International License, which permits use, sharing, adaptation, distribution and reproduction in any medium or format, as long as you give appropriate credit to the original author(s) and the source, provide a link to the Creative Commons licence, and indicate if changes were made. The images or other third party material in this article are included in the article's Creative Commons licence, unless indicated otherwise in a credit line to the material. If material is not included in the article's Creative Commons licence and your intended use is not permitted by statutory regulation or exceeds the permitted use, you will need to obtain permission directly from the copyright holder. To view a copy of this licence, visit http://creativecommons.org/licenses/by/4.0/.

\section{References}

1. 2017 North Carolina HIV/STD/Hepatitis Surveillance Report. https://epi.dph.ncdhhs.gov/cd/stds/figures/std17rpt_rev12142018. pdf. Accessed 1 July 2019.

2. North Carolina Public Health. Health Equity and HIV in North Carolina, 2017: Gay, bisexual, and other men who have sex with men. https://epi.dph.ncdhhs.gov/cd/stds/figures/factsheet_healt hequityHIV_MSM_2017.pdf. Accessed 1 July 2019.

3. North Carolina Public Health. HIV care outcomes in North Carolina, 2017. https://epi.dph.ncdhhs.gov/cd/stds/figures/factsheet HIV_care_outcomes_2017_rev2.pdf. Accessed 1 July 2019.

4. Magis-Rodriguez C, Lemp G, Hernandez MT, et al. Going North: Mexican migrants and their vulnerability to HIV. J Acquir Immune Defic Syndr. 2009;51(Suppl 1):S21-25.

5. Apostolopoulos Y, Sonmez S, Kronenfeld J, et al. STI/HIV risks for Mexican migrant laborers: exploratory ethnographies. J Immigr Minor Health. 2006;8:291-302.

6. Hirsch JS, Higgins J, Bentley ME, et al. The social constructions of sexuality: marital infidelity and sexually transmitted 
disease-HIV risk in a Mexican migrant community. Am J Public Health. 2002;92:1227-37.

7. Viadro CI, Earp JA. The sexual behavior of married Mexican immigrant men in North Carolina. Soc Sci Med. 2000;50:723-35.

8. Morales-Aleman MM, Sutton MY. Hispanics/Latinos and the HIV continuum of care in the Southern USA: a qualitative review of the literature, 2002-2013. AIDS Care. 2014;26:1592-604.

9. Vargas Bustamante A, Fang H, Garza J, et al. Variations in healthcare access and utilization among Mexican immigrants: the role of documentation status. J Immigr Minor Health. 2012;14:146-55.

10. Berk ML, Schur CL, Chavez LR, et al. Health care use among undocumented Latino immigrants. Health Aff. 2000;19:51-64.

11. Stefan MS, Blackwell JM, Crawford KM, et al. Patients' attitudes toward and factors predictive of human immunodeficiency virus testing of academic medical clinics. Am J Med Sci. 2010;340:264-7.

12. Mann L, Valera E, Hightow-Weidman LB, et al. Migration and HIV risk: life histories of Mexican-born men living with HIV in North Carolina. Cult Health Sex. 2014;16:820-34.

13. Barrington C, Gandhi A, Gill A, et al. Social networks, migration, and HIV testing among Latinos in a new immigrant destination: Insights from a qualitative study. Glob Public Health. 2018;13:1507-19.

14. Barrington C, Davis DA, Villa-Torres L, et al. Intersectionalities and the HIV continuum of care among gay Latino men living with HIV in North Carolina. Ethn Health. 2019;1:1-16.

15. Merino Y, Barrington C, Villa-Torres L, et al. Negotiating control of HIV: a qualitative analysis of HIV-positive Latino MSM in North Carolina. AIDS Behav. 2018;22:4048-56.

16. McCree DH, Walker $\mathrm{T}$, DiNenno E, et al. A programmatic approach to address increasing HIV diagnoses among Hispanic/ Latino MSM, 2010-2014. Prev Med. 2018;114:64-71.

17. Hightow-Weidman LB, Smith JC, Valera E, et al. Keeping them in "STYLE": finding, linking, and retaining young HIV-positive black and Latino men who have sex with men in care. AIDS Patient Care STDS. 2011;25:37-45.

18. Gardner LI, Metsch LR, Anderson-Mahoney P, et al. Efficacy of a brief case management intervention to link recently diagnosed HIV-infected persons to care. AIDS. 2005;19:423-31.

19. Gardner LI, Marks G, Craw J, et al. Demographic, psychological, and behavioral modifiers of the Antiretroviral Treatment Access Study (ARTAS) intervention. AIDS Patient Care STDS. 2009;23:735-42.

20. Demographic Facts You Need to Know: Hispanic/Latino, NC vs. U.S. https://www.ncdemography.org/wp-content/uploads/2019/ 09/20191011-Hispanic-Fact-Sheet.pdf. Accessed 3 Aug 2020.

21. Schiller NG, Basch L, Blanc-Szanton C. Transnationalism: a new analytic framework for understanding migration. Ann N Y Acad Sci. 1992;645:1-24.

22. Zimmerman C, Kiss L, Hossain M. Migration and health: a framework for 21 st century policy-making. PLoS Med. 2011;8:e1001034.

23. Parker R, Aggleton P. HIV and AIDS-related stigma and discrimination: a conceptual framework and implications for action. Soc Sci Med. 2003;57:13-24.
24. Saleebey D. The strengths perspective in social work practice: extensions and cautions. Soc Work. 1996;41:296-305.

25. Bradley KA, Rubinsky AD, Lapham GT, et al. Predictive validity of clinical AUDIT-C alcohol screening scores and changes in scores for three objective alcohol-related outcomes in a Veterans Affairs population. Addiction. 2016;111:1975-84.

26. Crawford EF, Fulton JJ, Swinkels CM, et al. Diagnostic efficiency of the AUDIT-C in U.S. veterans with military service since September 11, 2001. Drug Alcohol Depend. 2013;132:101-6.

27. Gonzalez P, Nunez A, Merz E, et al. Measurement properties of the Center for Epidemiologic Studies Depression Scale (CES-D 10): findings from HCHS/SOL. Psychol Assess. 2017;29:372-81.

28. Marin G, Gamba RJ. A New Measurement of acculturation for Hispanics: the bidemensional acculturation scale for Hispanics (BAS). Hisp J Behav Sci. 1996;18:297-316.

29. Schwartz SJ, Adamson L, Ferrer-Wreder L, et al. Identity status measurement across contexts: variations in measurement structure and mean levels among White American, Hispanic American, and Swedish emerging adults. J Pers Assess. 2006;86:61-76.

30. Zea MC, Asner-Self KK, Birman D, et al. The abbreviated multidimensional acculturation scale: empirical validation with two Latino/Latina samples. Cultur Divers Ethnic Minor Psychol. 2003;9:107-26.

31. Carrillo H. Sexual culture, structure, and change: a transnational framework for studies of Latino/a migration and HIV. HIV prevention with latinos: theory, research, and practice. New York: Oxford Scholarship; 2012. p. 41-61.

32. Villa-Torres L, Gonzalez-Vazquez T, Fleming PJ, et al. Transnationalism and health: a systematic literature review on the use of transnationalism in the study of the health practices and behaviors of migrants. Soc Sci Med. 2017;183:70-9.

33. Zhou YR, Coleman WD, Huang Y, et al. Exploring the intersections of transnationalism, sexuality and HIV risk. Cult Health Sex. 2017;19:645-52.

34. Rhodes SD, Hergenrather KC, Aronson RE, et al. Latino men who have sex with men and HIV in the rural south-eastern USA: findings from ethnographic in-depth interviews. Cult Health Sex. 2010;12:797-812.

35. Berk ML, Schur CL. The effect of fear on access to care among undocumented Latino immigrants. J Immigr Health. 2001;3:151-6.

36. Dang BN, Giordano TP, Kim JH. Sociocultural and structural barriers to care among undocumented Latino immigrants with HIV infection. J Immigr Minor Health. 2012;14:124-31.

37. Guilamo-Ramos V, Thimm-Kaiser M, Benzekri A, et al. The invisible US Hispanic/Latino HIV crisis: addressing gaps in the national response. Am J Public Health. 2020;110:27-31.

Publisher's Note Springer Nature remains neutral with regard to jurisdictional claims in published maps and institutional affiliations. 